\title{
L'impact Des Systèmes D'information Sur La Performance Des Chaines Logistiques: Une Revue De Littérature
}

\author{
Ouafae Zerouali Ouariti, P.E.S \\ Laila Zeroual, $\mathbf{P h D}$ \\ Equipe de Recherche: Economie de Transport, \\ Technologies de L'information et Logistique, \\ Ecole Nationale de Commerce et de Gestion d'AGADIR, \\ Université Ibn Zohr, Maroc
}

doi: 10.19044/esj.2017.v13n4p284 URL:http://dx.doi.org/10.19044/esj.2017.v13n4p284

\begin{abstract}
In the context of global competition which is characterized by a variety of products, short life cycles, and a competitive pressure between the supply chains, the cooperation and collaboration between companies has become very crucial. These collaborative relations improve the coordination of activities within the supply chains and have resulted to a higher performance through the use of information systems. These information systems have enabled it to face the rise in the complexity of the logistics sector which was born out of the information. To better position our contribution, we made a review of the literature and defined the variables and the hypotheses of this research work.
\end{abstract}

Keywords: Supply Chain, Logistics Information System (LIS), Logistics Performance, Collaboration.

\section{Résumé}

Dans un contexte de compétition mondialisée, caractérisé par une demande de plus en plus volatile et imprévisible, une variété croissante de produits, des cycles de vie qui raccourcissent, et une pression concurrentielle de plus en plus prononcée focalisée entre les chaînes logistiques et non plus entre les entreprises, la coopération et la collaboration entre les entreprises d'une même chaîne logistique devient primordial. Ces relations collaboratives améliorent la coordination des activités au sein des chaînes logistiques et permettent d'atteindre une plus grande performance grâce à l'utilisation des systèmes d'informations qui ont permis de faire face à la montée en complexité du secteur logistique dont tout naît de l'information. 
Pour mieux positionner notre contribution nous dressons une revue de littérature et nous définissons les variables et les hypothèses de notre travail de recherche.

Mots clés : La chaine logistique, Les systèmes d'information logistique, la performance logistique, La collaboration.

\section{Introduction}

Pour faire face aux fortes pressions de la concurrence mondiale, les chaines logistiques traversent les frontières et font appel à des relations avec un grand nombre de partenaires et de fournisseurs où leurs performance dépend de plus en plus de leur flexibilité et de leur capacité d'innover, cette innovation est tirée par la perspective d'une numérisation « de bout en bout » des flux informationnels, ce dernier a suscité la mise au point et le développement d'une série d'outils et de solutions technologiques dédiées à la gestion et aux échanges d'informations tout au long de la chaîne en permettant de partager des données correctes et précises. De cette manière, les membres de la chaîne sont capables d'accéder aux informations partagées et cette accessibilité peut être utilisée pour améliorer la conduite des opérations et au final améliorer la performance globale de la chaîne logistique.

Cette performance logistique constitue un rapport entre le service fourni au client et les moyens utilisés, elle consiste à assurer la satisfaction du client en lui livrant des produits de bonne qualité, au bon moment, en bonne quantité, et au bon endroit en consommant moins de ressources.

Notre recherche s'inscrit dans le cadre de la continuité des travaux de recherches, qui ont mis en lumière la relation entre les systèmes d'information et la performance des chaines logistiques

Dans cet article nous présentons dans un premier temps, une revue de littérature liée à la performance et surtout la performance logistique ainsi que les systèmes d'information logistique. Ensuite, les différents travaux qui analysent l'impact des SIL sur la performance des chaines logistiques. Enfin, nous discuterons quelques perspectives de notre recherche.

\section{La Performance Des Chaines Logistiques: Revue De Littérature}

Cette section a pour finalité de présenter le cadre théorique relatif à notre thématique. Dans un premier temps, nous commençons par la présentation de la notion logistique et de la performance, ensuite les composants de la performance logistique. 


\section{De La Logistique Au Supply Chain}

Le terme logistique vient d'un mot grec « Logistikos » qui signifie l'art du raisonnement et du calcul ou «Logisteuo » qui signifie administrer (Pons, 1996). Les institutions militaires ont utilisé ce terme pour définir l'activité qui réussit à combiner deux facteurs nécessaires dans la gestion des flux : l'espace et le temps. Dans ce contexte militaire, la logistique concerne tout ce qui est nécessaire (physiquement) pour permettre l'application sur le terrain des décisions stratégiques et tactiques (transports, stocks, fabrication, achats, manutention) (Pons, 1996). La logistique a donc été un sujet de réflexion intensif pour les grands chefs militaires.

Il existe de multiples définitions de la logistique, ce qui rend impossible actuellement d'en donner une définition acceptée par tous et dans tous les pays. Par ailleurs il est donc préférable d'en examiner les divers sens et dans des diverses dimensions, afin de pouvoir donner une approche de définition de la logistique et ne pas une définition précise.

La logistique dans l'entreprise est la fonction organisant la circulation des flux physique , d'information et financiers, autrement dit l'art de livrer, au moindre coût, le bon produit, au bon moment, au bon endroit, dans la quantité Just nécessaire, dans le bon conditionnement, avec les bons documents, précède, accompagné et suivie par les Bonnes informations.

Cette définition intègre d'une part la question de planification et du contrôle, et d'autre part les phases d'approvisionnements et de retour.

En 1948, le comité des définitions de l’American Marketing Association a proposé une première définition de la logistique : "Mouvement et manutention de marchandises du point de production au point de consommation ou d'utilisation. "

En (F.Magee, 1968) introduit la notion de flux : "la logistique est la technique de contrôle et de gestion des flux de matières et de produits, depuis leurs sources d'approvisionnement jusqu'à leurs points de consommation. "

Progressivement, les définitions s'affinent et expriment parallèlement la montée en puissance de cette fonction dans les entreprises. Heskett (1977) propose une analyse dynamique en termes de flux physiques et flux d'information, qui rend possible la coordination entre ces flux. Heskett a une grande influence, c'est pourquoi dès 1986, le Council of Logistics Management va revoir sa définition de 1972, d'une part en la simplifiant, d'autre part en y ajoutant la notion de flux d'information.

L'ASLOG à son tour dans sa définition insiste sur l'impératif de performance «La logistique est l'ensemble des activités ayant pour but la mise en place, au moindre coût, d'une quantité de produit, à l'endroit et au moment où une demande existe. La logistique concerne donc toutes les opérations déterminant le mouvement des produits tel que localisation des 
usines et entrepôts, approvisionnements, gestion physique des encours de fabrication, emballage, stockage et gestion des stocks, manutention et préparation des commandes, transports et tournées de livraison ».

La vision des chercheurs français est différente dans la mesure où ils accordent une importance au cycle de vie du produit .Ainsi, la norme NF-X 50-600 définit la logistique comme «la planification, l'exécution et la maitrise des mouvements et des mises en place des personnes ou des biens, et des activités de soutien liées à ces mouvements et mises en place, au sien d'un même système organisé pour atteindre des objectifs spécifiques ».

D'après ces définitions qui sont différentes mais complémentaires, on peut confirmer que la logistique est l'une des fonctions de l'entreprise, qui recouvre toujours les activités de transport, stockage et manutention. Elle tend, également, son domaine en amont vers l'achat et l'approvisionnement et en aval vers la gestion commerciale et la distribution (Yves Pimor \& Michel Fender,2008).

\section{Les Phases D’évolution De La Logistique}

Les recherches menées pendant la 2ème guerre mondiale servirent de point de départ à toutes les évolutions qui suivirent. La logistique passe alors par plusieurs phases d'évolution pour aboutir au concept de supply chain management. Nous décrivons successivement les différentes phases qu’a connues la logistique.

\subsection{1 la logistique séparée}

Du fait de l'émergence de la recherche d'optimisations opérationnelle dans les années 60 - 70 On observe dans un premier temps des optimisations disjointes (stocks, production..) visant à réduire le coût des opérations et à améliorer la circulation des flux sans chercher une optimisation globale des processus. On parle d'une logistique séparée

\begin{tabular}{|l|l|}
\hline Philosophie du management & Economie de masse \\
\hline Période de la demande & Unifiée \\
\hline Priorité du producteur & Quantité produite \\
\hline Choix du client & limité \\
\hline Relation entre producteur et client & Le producteur roi \\
\hline
\end{tabular}

Tableau 1. La Logistique de service ou Logistique séparée (1950 à 1975)

\subsection{2 la logistique intégrée}

La logique de la logistique séparée ne sera plus valable lorsque la production devient de plus en plus diversifiée, ce qui donne lieu à une logistique intégrée qui a pour définition l’intégration de deux ou plus de deux activités dans le but de planifier , mettre en œuvre et contrôler un flux 
efficient de matières premières, produit semi-finis... de leur point d’origine au point de consommation.

\begin{tabular}{|l|l|}
\hline Philosophie du management & Economie de singularité \\
\hline Période de la demande & Diversifiée \\
\hline Priorité du producteur & Qualité et la flexibilité \\
\hline Choix du client & Diversifié \\
\hline Relation entre producteur et client & Le client est roi \\
\hline
\end{tabular}

Tableau 2. La Logistique fonction ou Logistique Intégrée (de 1975 à 1990)

\subsection{3 la logistique coopérée}

Dans les années 90 l’approche de la logistique intégrée n’est plus suffisante pour obtenir l'avantage concurrentiel et pour répondre à un environnement concurrentiel en plein mutation, qui impose le passage à la micro-logistique qui suppose non seulement un passage obligé du système de circulation physique mais, également une collaboration entres acteurs de la chaine logistique.

On assiste à un décloisonnement, la notion de transversalité fait son apparition. L'entreprise passe ainsi à une démarche de recherche de l'efficacité et de la maîtrise de ses coûts.

A partir des années 90, la logistique va connaitre une importante évolution.

\begin{tabular}{|l|l|}
\hline Philosophie du management & Plein essor de l'économie de singularité \\
\hline Période de la demande & Personnalisée \\
\hline Priorité du producteur & Vitesse de réponse \\
\hline Choix du client & Personnalisé \\
\hline Relation entre producteur et client & Coopération entre le client et le producteur \\
\hline
\end{tabular}

Tableau 3. La Logistique Processus /Logistique coopéré (Les années 90)

Pour réussir à remplir ses objectifs les entreprises vont devoir aller au delà de la simple relation « marchande » et ainsi créer de réels partenariats avec les fournisseurs, clients et même concurrents. Ces coopérations vont participer à l'émergence des systèmes d'information logistiques. Ces outils et partenariats vont changer la donne en termes de concurrence. Là où avant nous avions une concurrence entre les producteurs aujourd'hui nous avons une concurrence entre les chaines logistiques « Supply chain ».

D'après (Lisa. M. Ellran, 2000), une SC est un réseau d'entreprises en interaction, dont l'objectif est de livrer un produit ou un service a l'utilisateur 
final, en intégrant les flux a partir des matières premières jusqu'a la livraison du produit fini.

\section{La Notion De La Performance}

Le concept de performance est omniprésent dans la littérature de gestion; de très nombreuses recherches s'intéressent à étudier l'impact d'un paramètre particulier sur la performance.

Nous trouvons un grand nombre de définitions relatives à la performance ce qui contribue à faire de la notion un « mot valise » qui a reçu de nombreuses acceptions (Saulquin \& Maupetit, 2004; Saulquin \& Schier, 2007, p.59). Nous illustrons dans le tableau ci-dessous quelques unes.

\begin{tabular}{|l|l|}
\hline Albanes,1978 & $\begin{array}{l}\text { «la performance est la raison des postes de gestion, } \\
\text { elle implique l'efficience et l'efficacité ». }\end{array}$ \\
\hline Machesnay,1991 & $\begin{array}{l}\text { «la performance de l'entreprise peut se définir } \\
\text { comme le degré de réalisation du but recherché ». }\end{array}$ \\
\hline Berrah, 2002 & $\begin{array}{l}\text { « Une entreprise performante est une entreprise qui } \\
\text { atteint les objectifs qu'elle annonce ». }\end{array}$ \\
\hline Stefan, 2004 & $\begin{array}{l}\text { «la performance est l'ensemble de mesures utilisées } \\
\text { dans la quantification de l'efficience et de l'efficacité } \\
\text { d'une action ». }\end{array}$ \\
\hline Shepherd \& Günter, 2006 & $\begin{array}{l}\text { «la performance est déclinée en termes d'efficacité, } \\
\text { de pertinence et d'efficience ». }\end{array}$ \\
\hline Pichot, 2006 & $\begin{array}{l}\text { "la performance est l'efficience et l'efficacité d'une } \\
\text { organisation à réaliser ses objectifs ». }\end{array}$ \\
\hline Notat, 2007 & $\begin{array}{l}\text { « un constat officiel enregistrant un résultat accompli } \\
\text { à un instant t, toujours en référence à un contexte, à } \\
\text { un objectif et un résultat attendu, et ce quel que soit } \\
\text { le domaine ». }\end{array}$ \\
\hline Armstrong, 2009 & $\begin{array}{l}\text { "C'est un processus systématique qui vise à } \\
\text { améliorer la performance organisationnelle en } \\
\text { développant la performance des individus et des } \\
\text { équipes ». }\end{array}$ \\
\hline «le résultat d'une action ». \\
\hline Marion et al., 2012
\end{tabular}

Tableau 4. Définitions de la performance

Ces définitions citées précédemment, montrent que la performance est associée à l'efficacité et à l'efficience: Efficacité rapport entre les résultats obtenus et les objectifs fixés. Efficience rapport entre les moyens et les efforts.

\section{La Performance Logistique}

On se basant sur le rapport d'AFNOR, 2015 nous définissons la performance logistique comme une mesure de rapport entre le service fourni au client et les moyens consommés. Une logistique performante assure la 
satisfaction du client en consommant moins de ressources, cependant la performance d'un acteur au sein de sa chaîne logistique apparait à travers la satisfaction d'un ensemble d'objectifs inhérents à la stratégie choisie. Ces objectifs sont établis sur plusieurs horizons et on parle alors d'objectifs stratégiques, tactiques et opérationnels.

Ceci implique la déclinaison de la performance sur ces trois mêmes niveaux décisionnels (Berrah, 2002) et pour évaluer le degré d’atteinte de chaque objectif, une entreprise a alors recours à la mesure de ses performances (Supply Chain Meter, 2012) en vue de: "Se situer par rapport aux objectifs fixés et comprendre les écarts, Se comparer en interne comme en externe (benchmark), S’améliorer individuellement, conjointement, collectivement, Concevoir des circuits logistiques plus fiables, optimisés, réactifs et respectueux de l'environnement ».Pour faire, elle s'appuie sur un ensemble d'indicateurs de performance ou système d'indicateurs.

Les indicateurs de performances: Selon (Lorino, 2001) et (Bouquin, 2004) un indicateur de performance est associé à une " action à piloter ». Pourtant les indicateurs logistiques se résument à faire « mieux que les autres, plus vite, moins cher, plus écologique », en mettant l'accent sur quatre leviers clés, soient la fiabilité, l’efficience, la réactivité et le respect de l'environnement.

— La fiabilité logistique. (Thierry Jouenne, 2011) «la fiabilité consiste en la capacité de répondre à la demande du client selon un niveau de service fixé ».

Les indicateurs susceptibles de mesurer cette fiabilité logistique sont le taux de service fournisseurs, le taux de service client, taux de fiabilité des prévisions, le taux de service transport, le taux de réclamations, le taux d'absentéisme du personnel, etc.

- L'efficience logistique, rapport entre efficacité et coût. Il vise à atteindre un objectif avec le minimum de moyens engagés.

Les indicateurs susceptibles de mesurer cette efficience logistique sont coût de possession de stock, coût total d'achat, coût de fabrication, coût de transport, etc.

- La réactivité logistique, capacité de s’adapter rapidement aux fluctuations de la demande.

Les indicateurs susceptibles de mesurer cette réactivité logistique: rotation des stocks, time-to-market, vitesse d'écoulement des produits, ratio de tension des flux, cycle order-to-cash, cycle cash-to-cash, temps de cycle, de transit, d'attente, etc.

- Le volet de respect de l'environnement, à travers l'adoption de pratiques "vertes" tels que le transport multimodal, mutualisation des 
infrastructures et du transport, logistique inverse ainsi que le recyclage des produits.

Pour les indicateurs éco-logistiques, le pourcentage de la consommation d’énergie, l'émission de gaz à effet de serre les tonnes de CO2 émises par les activités logistiques, etc.

\section{Les Systèmes D'information}

Selon (James A. O' Brien, 1995), un système d'information est un ensemble de personnes, de procédures, et de ressources qui recueillent l'information, la transforment et la distribuent au sein d'une organisation. Selon (Robert Reix, 2004), il s'agit de l'association de ressources humaines, matérielles et logicielles destinées à recueillir, formaliser, archiver, parcourir, associer et diffuser l'information dans cette même organisation ».

Les systèmes d'information sont apparus dans les fonctions d'entreprises dont les métiers qui nécessitent la manipulation de grandes quantités de données telles que la finance, ensuite dans les fonctions qui nécessitent beaucoup de planification et de rigueur, comme la logistique et le management des ressources humaines qui ont été dotés d'outils informatiques. Enfin les fonctions qui travaillent en réseau, comme le marketing et les achats, ont adopté des SI à la fois intra et inter organisationnels. Les systèmes d'information aident les managers à piloter des processus, à analyser des performances, à anticiper des évolutions et à prendre des décisions. Les informations qui leur sont fournies doivent donc être particulièrement actualisées et fiables.

\section{Les Systémes D’information Logistiques}

Au sein des organisations, les besoins d'information sont différents pour chaque niveau de management, tant au niveau du fond que de la forme de l'information.Il faut donc adapter l'information et pouvoir la modifier en fonction de l'environnement et du contexte organisationnel.

Le fonctionnement d'une chaîne logistique est basé sur la circulation des flux informationnels en interne et en externe. Cette circulation est rendue possible grâce à l'utilisation des SIL, qui représentent la colonne vertébrale de la fonction logistique (Wood et al., 2015).

Dans une chaine logistique les systèmes d'information sont classés en trois catégories principales et complémentaires:

Les SI « entreprise étendue »;

Les SI intégrateur ;

Les SI facilitateurs; 


\begin{tabular}{|c|c|c|c|}
\hline Catégorie & $\begin{array}{c}\text { Système } \\
\text { d'information }\end{array}$ & Caractéristiques & Auteurs \\
\hline $\begin{array}{l}\text { Les SI « } \\
\text { entreprise } \\
\text { étendue " }\end{array}$ & $\begin{array}{c}\text { ERP«Enterprise } \\
\text { Resource } \\
\text { Planning» }\end{array}$ & $\begin{array}{l}\text {-Proposent, au sein d'une même } \\
\text { architecture une base de données } \\
\text { unique, une intégration des données } \\
\text { transactionnelles mobilisées par les } \\
\text { différentes fonctions de gestion de } \\
\text { l'entreprise, } \\
\text { - Se caractérisent par des fonctions } \\
\text { standards. } \\
\text {-Assurent la standardisation des } \\
\text { échanges de données au sein } \\
\text { de l'organisation et inter-organisation }\end{array}$ & $\begin{array}{c}\text { (P. M. } \\
\text { Swafford, S. } \\
\text { Ghosh, \& N. } \\
\text { Murthy, 2008) }\end{array}$ \\
\hline $\begin{array}{l}\text { Les SI } \\
\text { qualifiés " } \\
\text { Intégrateurs } \\
\text { " }\end{array}$ & $\begin{array}{l}\text { APS « Supply } \\
\text { Chain Planning } \\
\text { », (Advanced } \\
\text { Planning } \\
\text { System) }\end{array}$ & $\begin{array}{l}\text { - Permettent de planifier l'ensemble } \\
\text { des processus de la chaîne logistique. } \\
\text { Ses principales fonctions sont : la } \\
\text { planification de la production, des } \\
\text { transports et les prévisions des } \\
\text { ventes. } \\
\text {-Permettent de gérer l'exécution des } \\
\text { opérations logistiques (Achats, } \\
\text { approvisionnements, gestion de la } \\
\text { production, gestion des stocks } \\
\text { matières, en -cours, produits finis, } \\
\text { gestion des opérations de transport). } \\
\text { Dans les SCE on retrouve: } \\
\text {-les MES pour la fabrication } \\
\text { (ManufacturingExecution System) } \\
\text {-les WMS pour la gestion des } \\
\text { entrepôts (Warehouse Management } \\
\text { System) -les TMS pour la gestion du transport } \\
\text {-les OMS pour gérer les ordres et les } \\
\text { ventes }\end{array}$ & $\begin{array}{l}\text { (P.-A. Millet \& } \\
\text { V. Botta- } \\
\text { Genoulaz, } \\
\text { 2011) }\end{array}$ \\
\hline $\begin{array}{l}\text { Les SI } \\
\text { qualifiés " } \\
\text { facilitateurs } \\
"\end{array}$ & $\begin{array}{c}\text { EDI } \\
\text { «Échanges de } \\
\text { Données } \\
\text { Informatisé » }\end{array}$ & $\begin{array}{l}\text {-Permettent d'accélérer la diffusion et } \\
\text { le traitement des informations entre } \\
\text { les partenaires. } \\
\text {-Consistent à échanger entre } \\
\text { (Clients/Fournisseurs) et par voie } \\
\text { électronique (Ordinateurs et réseaux } \\
\text { de télécommunication) des } \\
\text { informations structurées, référencées } \\
\text { dans des documents normalisés } \\
\text { (commandes, bons de livraison, } \\
\text { factures, états de stock, etc...) }\end{array}$ & $\begin{array}{l}\text { (E. W. T. Ngai, } \\
\text { K.-H. Lai, \& T. } \\
\text { C. E. Cheng, } \\
\text { 2008) } \\
\text { (ÉRIC- } \\
\text { Brousseau, } 199 \\
\text { 1) }\end{array}$ \\
\hline
\end{tabular}

Tableau 5. Les systèmes d'information logistique

Selon (Chafik \& Boubker, 2016) "le fonctionnement de la chaîne logistique est fondé sur la coexistence de deux types d'intégration 
complémentaire: l'intégration inter-fonctionnelle et celle interorganisationnelle"La première est basée sur l'intégration de l'ensemble des processus logistiques depuis l'amont jusqu'au l'aval (Tyndall et al., 1998). Alors que la deuxième est construite autour d'une série de relations entre les entreprises partenaires qui partagent mutuellement les informations, les risques et les récompenses qui amènent à l'avantage compétitif (Cooper \& Ellram, 1993)".

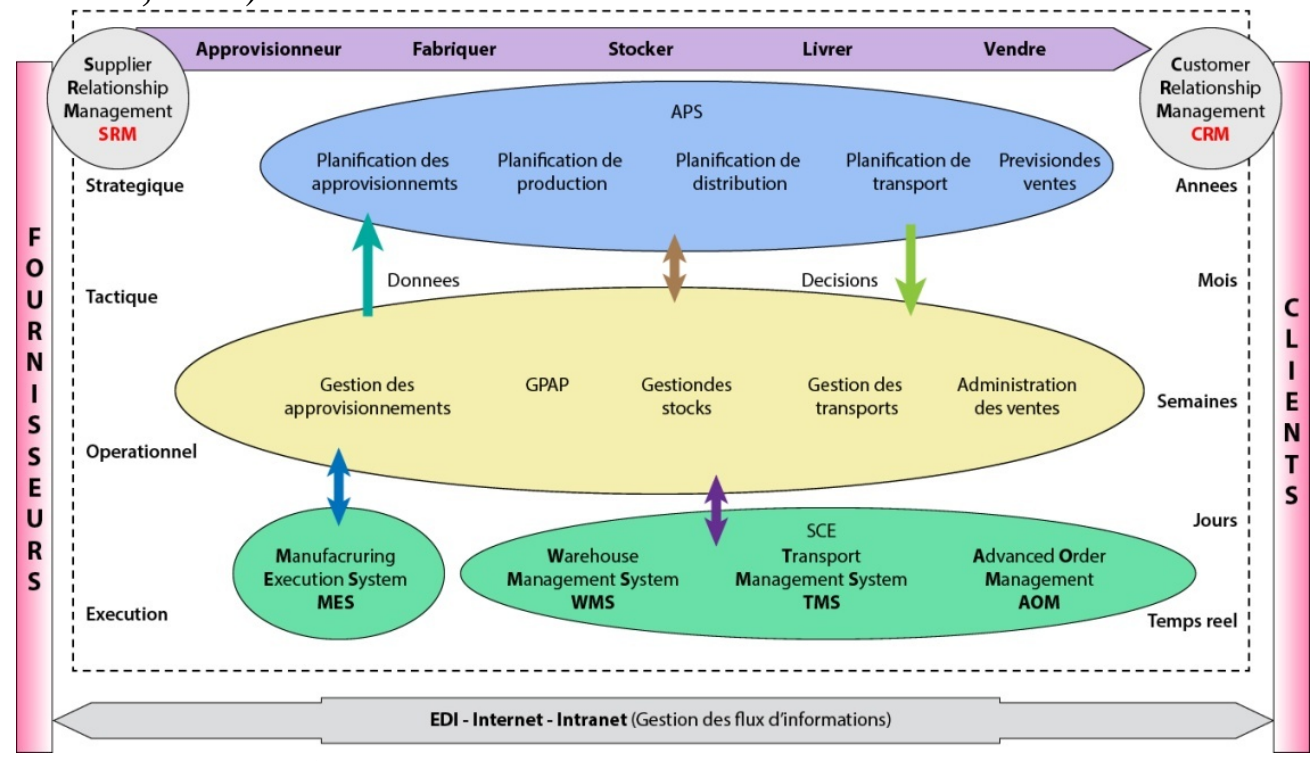

Figure 1. Couverture des SIL dans une Supply Chain

\section{Problématique De Recherche}

La démarche logistique est fondée sur un principe de pilotage des flux physiques par les flux d'information. À ce titre, les systèmes d'information ont toujours occupé, une partie importante de la recherche en gestion de la chaîne logistique portant sur l'apport du partage d'informations au niveau de la coordination entre les partenaires de la chaîne logistique (Chen, 2003).

Notre problématique de recherche vise à montrer l'impact des systèmes d'information sur la performance des chaines logistiques. Notre problématique générale qui représente l'ossature de notre recherche peut s'énoncer comme suit: Dans quelle mesure l'usage des systèmes d'information influence-t-il la performance des chaines logistiques?

Pour répondre à cette problématique, une analyse de la littérature est primordial, pour recenser les différents variables et critéres retenus par les chercheurs en sciences de gestion et particulièrement en logistique et en management des systèmes d'information. 


\section{Analyse De La Littérature}

Si la logistique s’intéresse principalement aux flux de marchandises c'est grâce aux flux d’informations qu'elles arrivent à les piloter.Le point commun entre les travaux qu'on va présenter ci-dessous c’est qu’ils cherchent à améliorer la performance globale de la chaîne logistique.

L'utilisation des systèmes d'information, le partage et l'échange des informations, la communication inter et intra-organisationnelles, la coopération et la collaboration intra- et inter-organisationnelle forment des facteurs indispensables pour la performance de la chaîne logistique.

Sanders (2007) proposent un modèle de relation entre l'usage des technologies de l'information, la collaboration et la performance organisationnelle, qui rend possible la collaboration entre les partenaires de la chaine en temps réel. Cette étude a confirmé l'impact positif de l'usage des technologies sur la performance de la chaine et sur la collaboration intra et inter-organisationnelle. Anderson et Narus en (1990) ont travaillé aussi sur la collaboration inter-organisationnelle et ont montré que l'échange d'informations contribue à une meilleure coordination des actions des partenaires leur permettant d'atteindre plus facilement les objectifs fixés.

Zhou et Benton (2007) et Cheng (2013) proposent la standardisation de tous les processus de la chaîne logistique. Permettant la diminution des incertitudes, ce qui débouche un angle de réactivité à la chaîne logistique. Sur la même angle Sink et Langley (1997) ont montré que la continuité des flux d'information, tout comme celle des flux physiques, ne doit pas être compromise afin de réduire l'incertitude, de minimiser le risque de confusion ou de conflit et d'assurer l'efficacité du processus.

De nombreux auteurs s’intéressaient à intégrer dans leurs études le volet de la confiance tel que. Klein (2007) a mis l'accent sur la relation entre les fournisseurs et les clients en vue de créer un climat collaboratif. Cette relation est basée principalement sur la confiance qui joue un rôle primordial dans l'évolution des relations inter-organisationnelles et est considérée comme le composant noyau et facteur clé de succès des chaines logistique. Ganesan (1994), Gulati (1995), et Paulraj et al. (2008) considèrent la communication inter-organisationnelle comme un facteur critique au niveau de la collaboration entre les acteurs. Yang et al (2008) s’intéressaient à l'engagement relationnel et la confiance envers les fournisseurs. Kwon et Suh (2005) affirment quele partage d'information et la confiance entre les partenaires sont nécessaire pour avoir une chaine logistique efficace. Chen et al. (2011) ont étudié le rôle du partage de l'information, la qualité de l'information, et la disponibilité de l'information dans le développement de la confiance et de l'engagement dans les relations des partenaires d'une chaine logistique 
Zhou et Benton (2007) se basent sur trois aspects de partage d'information (la technologie de support de partage d'information, le contenu de l'information et la qualité de l’information) .Ce travail a montré que le partage d'information, avec ses trois aspects, et le dynamisme de la chaîne logistique influent fortement sur l'efficacité des pratiques de la chaîne. En cherchant toujours à améliorer la performance globale de la chaine logistique. Chowa et al. (2008) ajoutent d'autres aspects comme la communication, le partage d'information, l'intégration, la structure de la chaîne logistique, influent positivement sur la performance globale de la chaîne logistique.

Chan, Au, \& Chan (2006) expliquent que l'absence d'un système d'information a un impact négatif sur la performance et la pérennité de la chaine logistique et confirment que le partage de l'information en début de chaîne (le coût de production, les délais d’opération et la capacité disponible ...) peut influencer considérablement l'efficacité du système. Simatupang et al. (2004) ont conclu que la collaboration entre les partenaires dans le partage des informations facilite la synchronisation de décision entre ces partenaires, ce qui contribue à une performance significative.

Hsu et al. (2008) se sont intéressés au partage d'information qui permet d'améliorer les relations entre acheteur-fournisseur. Vereecke et Muylle (2006) expliquent que le partage d'information et la collaboration des acteurs d'une même chaîne logistique se traduit par l'amélioration de la performance globale de cette chaîne.

Nyaga et al. (2010) montrent que les entreprises sont en train de mettre en place des relations collaboratives avec leurs partenaires de la chaîne logistique afin d'atteindre à la fois : l'efficience, la flexibilité et l'avantage concurrentiel.

Le but de l'étude de Nath et permanent (2010) est d'identifier les systèmes d'information utilisés dans une chaîne logistique et d'analyser la littérature pour identifier les facteurs clés de sa réussite. Cheng (2013), Jain, Wadhwa, et Deshmukh (2009) ont notamment expliqué que le partage d'information de manière significative affecte la réduction des coûts logistique, et l'atteinte de l'avantage concurrentiel. Chengalur- Smith et al. (2012) ont conclu que le partage de l'information et l'optimisation du système d'entreprise peuvent fournir des avantages (à savoir, les intérêts commerciaux partagés entre les partenaires de la chaîne logistique).

Zhou et al. (2014), montrent que les partenaires ont besoin d'aligner les pratiques de la chaîne logistique avec la qualité de leurs informations afin d'obtenir une meilleure performance globale, confirmant les travaux de Picard et Tang-Taye (2000) ont précisé que l'objectif des systèmes d'information est de rendre le pilotage de la chaîne logistique globale encore plus efficace. 


\section{Les Variables Retenues De La Littérature}

On se basant sur les conclusions des travaux théoriques sur les systèmes d'information en relation avec la performance des chaines logistiques, nous avons conçu des hypothèses de recherche traduisant l'influence positive ou négative de nos variables retenues de la littérature sur la performance des chaines logistiques.

\begin{tabular}{|c|c|c|}
\hline Variables & Les hypothèses & Auteurs \\
\hline $\begin{array}{l}\text { La qualité de } \\
\text { l’information } \\
\text { La qualité du système } \\
\text { d’information }\end{array}$ & $\begin{array}{c}\text { H1 : La qualité des systèmes } \\
\text { d'information influence positivement la } \\
\text { qualité des informations. } \\
\text { H2 : La qualité des informations influence } \\
\text { positivement la qualité des systèmes } \\
\text { d'information. } \\
\text { H3 : La qualité des systèmes } \\
\text { d'information influence positivement la } \\
\text { satisfaction. } \\
\text { H4 : La qualité des systèmes } \\
\text { d'information influence positivement les } \\
\text { utilisations. } \\
\text { H5 : La qualité des informations influence } \\
\text { positivement la satisfaction des } \\
\text { utilisateurs. } \\
\text { H6 : La qualité des systèmes } \\
\text { d’information influence positivement la } \\
\text { satisfaction. }\end{array}$ & $\begin{array}{l}\text { Zhou \& Benton } \\
\text { (2007), } \\
\text { Zhou et al. (2014), } \\
\text { Chantal. M (2011), } \\
\text { Marie. B. Yves. G. } \\
\text { (2011), Chen et al. } \\
\text { (2011) }\end{array}$ \\
\hline $\begin{array}{l}\text { L'utilisation et la } \\
\text { satisfaction }\end{array}$ & $\begin{array}{l}\text { H7 : il existe une relation positive entre } \\
\text { l'utilisation et la satisfaction. }\end{array}$ & $\begin{array}{l}\text { S. Pettera \& E.R. } \\
\text { McLean (2009), J. } \\
\text { Iivari (2005). } \\
\end{array}$ \\
\hline La confiance & $\begin{array}{l}\text { H8 : La confiance inter-organisationnelle } \\
\text { influence positivement l'utilisation des } \\
\text { systèmes d'information. }\end{array}$ & $\begin{array}{c}\text { Klein (2007), } \\
\text { Ganesan (1994), } \\
\text { Gulati (1995), Paulraj } \\
\text { et al. (2008), Kwon \& } \\
\text { Suh (2008), Chen et } \\
\text { al. (2011). }\end{array}$ \\
\hline $\begin{array}{l}\text { La collaboration } \\
\text { inter- } \\
\text { organisationnelle }\end{array}$ & $\begin{array}{c}\text { H9 : La satisfaction des utilisateurs } \\
\text { influence positivement la collaboration } \\
\text { inter-organisationnelle. } \\
\text { H10 : L’utilisation influence positivement } \\
\text { la collaboration inter-organisationnelle. } \\
\text { H11 : La collaboration inter- } \\
\text { organisationnelle influence positivement } \\
\text { la performance de la chaine logistique }\end{array}$ & $\begin{array}{c}\text { Sanders (2007), } \\
\text { Anderson \& Narud } \\
\text { (1990), Klein (2007), } \\
\text { Sanders (2007), } \\
\text { Anderson \& Narus } \\
\text { (1990), Klein (2007), } \\
\text { Chowa et al. (2008), } \\
\text { Simatupang et al. } \\
\text { (2004), Vereecke \& } \\
\text { Muylle (2006), Nath } \\
\text { \& permanent (2010). }\end{array}$ \\
\hline
\end{tabular}

Tableau 6. Les variables et les hypothèses de notre recherche 
Généralement, le jugement des variables à retenir pour évaluer les gains logistiques apportées par l'utilisation des systèmes d'information, dépend de plusieurs éléments, citons à ce niveau : le contexte d'étude, le type des systèmes d'information utilisé, etc. (W. H. Delone \& E. R. McLean, 2003).

\section{Conclusion et Perspective de Recherche}

Sur la base de l'analyse de la revue de littérature, nous avons essayé d'identifier et de sélectionner l'ensemble des variables retenues par les chercheurs pour mesurer les bénéfices apportés par l'utilisation des systèmes d'information.

Les prochaines étapes de notre recherche consisteront à opérationnaliser ses variables dans un contexte marocain et tester lesdites hypothèses de travail, en vu de trouver des réponses concluantes à notre questionnement, via une approche qualitative exploratoire hybride, en se basant sur les étapes du paradigme de Churchill.

\section{References:}

1. Anderson, J.C. \& Narus J.A. (1990). « A Model of Distribution Firm and Manufacturer Firm Working Partnerships », Journal of Marketing, vol. 54, p. 42-58.

2. Boubker, O. \& Chafik, K. (2016). Understanding The Role Of Information Systems In Improving Performance And Maturity Of Logistic Processes: Qualitative Study In Six Companies Of The Automotive And Aeronautical Industry In Morocco. European Scientific Journal, ESJ, 12 (28).

3. Bouquin H. (2004). Le Contrôle de gestion, PUF, Gème édition.

4. Chowa, W. S., Madu, C. N., Kuei, C.-H., Lu, M. H., Chinho, \& L., Tseng, H. (2008). Supply chain management in the US and Taiwan: An empirical study. The International Journal of Management Science, 36 (5), pp. 665-679.

5. Colin, Monica, Galindo, \& Raquel (2015). Information and Communication Technology as a Key Strategy for Efficient Supply Chain Management in Manufacturing SMEs, Procedia Computer Science, VOL.55.p, 833-842.

6. Florence Rodhain, Bernard Fallery, Aur_elie Girard, \& Sylvie Desq (2010). Une histoire de la recherche en Systèmes d'Information, à travers 30 trente ans de publications. Entreprises et Histoire, Eska , p.78-97. <hal-00773589>.

7. Ganesan, S. (1994). Determinants of long-tem orientation in buyer seller relationships. Journal of Marketing, 58, pp. 50- 69. 
8. Gulati, R. (1995). Does familiarity breed trust? the implications of repeated ties for contractual choice alliance, The Academy of Management Journal.

9. Gunasekaran \& E.W.T. Ngai (2004). Information systems in supply chain integration and management, European Journal of Operational Research ,VOL.159 .p,269-295.

10. Heskett (1973). «Sweeping changes in distribution » Harvard Business Review, Vol,51, Issue n². March-April, P, 123-132.

11. Hudnurkar, ManojJakhar, Suresh Rathod, \& Urvashi (2014). Factors Affecting Collaboration in Supply Chain: A Literature Review, Procedia - Social and Behavioral Sciences,VOl.133,p. 189-202.

12. J.F. Magess (1968). Industrial Logistics. Analysis and Management of Physical Supply and Distribution Systems, New York, McGrawHill.

13. J. L. Heskett (1977). « Logistics : essential to strategy », Harvard Business Review , nov.-déc. traduiten 1978 par « La logistique, élément clé de la stratégie », Harvard-L'Expansion, $\mathrm{n}^{\circ} 8$.

14. K. Evrard-Samuel, S. Ruel, \& Alain Spalanzani (2011). Systèmes d'information et résilience des chaines logistiques globales : Proposition d'un écosystème informationnel. Cahier de recherche $d u$ CERAG 2011-03 E5. 2011. <halshs-00659359>.

15. Kwon, I. W. G., \& Suh, T. (2005). Trust, commitment and relationships in supply chain management: a path analysis. Supply Chain Management: An International Journal, 10(1), 26-33.

16. L. Berrah (2002). L'indicateur de performance : concepts et applications, Cépaduès.

17. Lee, H., K. C. So \& C. Tang (2000). The value of information sharing in a two level supply chain. Management Science, 46, 626643.

18. Les défis logistiques des PME en MP-Thierry Jounne (2011). CFPIM , Supply Chain Masters, Toulouse, 7avril.

19. LORINO, P. (2001). Méthodes et pratiques de la performance, Editions d'Organisation, 3ème Edition, Paris, France.

20. Marinagi, C.Trivellas, \& P.Reklitis (2015). Information Quality and Supply Chain Performance: The Mediating Role of Information Sharing, Procedia - Social and Behavioral Sciences,VOL.175,p. 473479.

21. Montoya-Torres, Jairo R. Ortiz-Vargas, \& Diego A (2014). Collaboration and information sharing in dyadic supply chains: A literature review over the period 2000-2012, EstudiosGerenciales, VOl,30.p,343-354. 
22. O’Brien James A. (1995). Les Systèmes d'Information de gestion, De Boeck Université.

23. O’Brien James A. (1995). Les Systèmes d'Information de gestion, De Boeck Université.

24. Omar Sakka, Valerie Botta-Genoulaz, \& Lorraine Trilling (2009). Modélisation des facteurs influençant la performance de la chaine logistique. INSA de Lyon, LIESP. 7 pages. <hal-00389129>.

25. Paulraj, A., Lado, A. A., \& Chen, I. J., (2008). Interorganizational communication as a r!pelational competency: Antecedents and performance outcomes in collaborative buyer-supplier relationships. Journal of Operations Management, 26, pp. 45-64.

26. Picard P., \& Tang-Taye J.P. (2000). «Impact de la mise en œuvre d'une alliance logistique sur les systèmes d'information des acteurs», Les Troisièmes Rencontres Internationales de la Recherche en Logistique, Trois-Rivières, 9-11 mai.

27. Reix Robert (2004). Systèmes d'Information et management des organisations, Vuibert, 5 édition.

28. Sanders, N. R., (2007). An empirical study of the impact of ebusiness technologies on organizational collaboration and performance. Journal of Operations Management, 25, pp. 1332-1347.

29. Saulquin J.Y., \& Maupett C. (2004). EVA, performance et évaluation bancaire, Journée de recherche CERMAT" La performance : de la mesure à l'action ". 15 janvier 2004.

30. ShahramGilaninia (2011). The Impact of Information Technology Application on Supply Chain Performance, interdisciplinary journal of contemporary research in business, VOL. 3, N 8, P.489-496.

31. Simatupang, T. M., Wright, A. C. \& Sridharan, R. (2004). Applying the theory of constraints to supply chain collaboration." Supply Chain Management: An International Journal 9 (1), 57-70.

32. Sink H.L. \& Langley C.J. (1997). «A Managerial Framework for the Acquisition of Third Party Logistics Services », Journal of Business Logistics, vol. 18, $\mathrm{n}^{\circ} 2$, p. 163-189.

33. Tseng, Ming-Lang Wu, Kuo-JuiNguyen, \& ThiThoa (2011). Information technology in supply chain management: a case study, Procedia - Social and Behavioral Sciences,VOL.25,p. 257-272.

34. Vereecke A., \& Muylle S. (2006). Performance improvement through supply chain collaboration in Europe, International Journal of Operations \& Production Management, Vol. 26, $\mathrm{n}^{\circ} 11$, pp. 11761198.

35. W. Y. Zhang \& Z. J. Gong (2014). D.Q. Journal of Applied Research and technology,VOL,12,p.182-191. 
36. Yang, J., Wang, J., Wong, C. Y., \& Lai., K.-H. (2008). Relational stability and alliance performance in supply chain. The International Journal of Management Science, Omega 36 pp. 600 - 608.

37. Yves Pimor \& Michel Fender (2008). Logistique: production, distribution, soutien : édition 5 page4.

38. Zainah Abdullah (2014). The Effect of Trust and Information Sharing on Relationship Commitment in Supply Chain Management, Procedia - Social and Behavioral Sciences,VOL.130 ,p.266 - 272.

39. ZEROULI OUARITI Ouafae, \& MOUNIR Younes (2012). Study of information sharing in the supply chain of a supplier and a distributor, International Journal of Research in Management \& Technology, Vol. 2, No. 1,P.29-340.

40. Zhao, X., Xie, J., \& Leung, J., (2002). The Impact of Forecasting Model Selection on the Value of Information Sharing in a Supply Chain. European Journal of Operational Research, 142(2), 321-344.

41. Zhou, H. \& Benton, W. C. J., (2007). Supply chain practice and information sharing. Journal of Operations Management, 25 pp. 1348-1365.

42. Zsidism, G.A., Jun, M., \& Adams, L.L. (2000). The relationship between information technology and service quality in the dualdirection supply chain: A case study approach. International Journal of Service Industry Management, 11 ,312-328. 